\title{
Design and Experimental Study on Divergent Magnetic Fluid Seal with Large Clearance and Dual Magnetic Sources
}

\author{
Xiaolong Yang $\mathbb{D},{ }^{1}$ Yunyun Song $\mathbb{D},{ }^{1,2}$ Peng Sun, ${ }^{1}$ and You $\mathrm{Li}^{1}$ \\ ${ }^{1}$ School of Mechanical and Automotive Engineering, Guangxi University of Science and Technology, Liuzhou 545006, China \\ ${ }^{2}$ Guangxi Earthmoving Machinery Collaborative Innovation Center, Liuzhou 545006, China \\ Correspondence should be addressed to Xiaolong Yang; yangxiaolong@gxust.edu.cn
}

Received 1 September 2021; Accepted 5 January 2022; Published 30 January 2022

Academic Editor: Guoqiang Xie

Copyright $(2022$ Xiaolong Yang et al. This is an open access article distributed under the Creative Commons Attribution License, which permits unrestricted use, distribution, and reproduction in any medium, provided the original work is properly cited.

In order to solve the leakage problem of rotating machinery under the condition of large clearance, a new type of divergent magnetic fluid sealing device with secondary magnetic source was designed. The magnetic field distribution in the clearance of the magnetic fluid seal was simulated by the finite element method of magnetic field, and the theoretical value of the sealing pressure resistance was calculated according to the pressure resistance theory of the divergent magnetic fluid seal. The influences of the volume of magnetic fluid injection, the number of teeth, the clearance, and the eccentricity of the rotating shaft on the pressure resistance abilities of divergent magnetic fluid seal were studied by experimental method and compared with the pressure resistance experimental value of ordinary magnetic fluid seal. The results showed that when the injection volume of magnetic fluid is more than $3 \mathrm{ml}$, the sealing pressure resistance ability of the magnetic fluid does increase greatly and tends to be stable gradually. The saturated magnetic fluid injection volume of divergent magnetic fluid seal with large clearance is $3 \mathrm{ml}$. With the increase of radial teeth, the pressure resistance ability of magnetic fluid seal first increases and then decreases. With the increase of axial teeth, the pressure resistance ability of magnetic fluid seal also increases. With the increase of radial clearance, the pressure resistance value of magnetic fluid seal first increases and then decreases. With the increase of axial clearance, the pressure resistance value of magnetic fluid seal decreases. The pressure resistance ability of divergent magnetic fluid seal is significantly higher than that of ordinary magnetic fluid seal.

\section{Introduction}

Magnetic fluid seal plays a vital role in engineering because they have the advantages of simple structure, high reliability, and long service life [1-3]. In the field of magnetic fluid seal, the clearance that is less than or equal to $0.3 \mathrm{~mm}$ is small clearance, and the clearance which is larger than $0.3 \mathrm{~mm}$ is called large clearance. The magnetic fluid seal has high reliability under the condition of small clearance, but the larger seal clearance $[4,5]$ will reduce the pressure resistance ability of magnetic fluid seal, so how to improve the pressure resistance ability of magnetic fluid seal under large clearance is a difficult problem at the moment. Zhao et al. carried out numerical analysis on the magnetic fluid seal with small clearance [6] and found that when the material and structure are the same, the magnetic fluid sealing ability decreases with the increase of the sealing clearance. Szczech carried out an experimental study on the pressure distribution mechanism between stages of the magnetic fluid seal [7]. The abovementioned people mainly studied the magnetic fluid seal with small clearance. In 2018, Saurabh et al. studied the static and dynamic sealing performance of magnetic fluid seal with radial sealing clearance height of $0.7 \mathrm{~mm}$ and studied the influence of magnetic source number on the performance of magnetic fluid seal [8]. In 2020, Parekh et al. designed and tested the performance of bipolar magnetic fluid seal under variable speed and radial clearance $(0.3-0.5 \mathrm{~mm})$ [9]. In order to improve the pressure resistance ability, Yang et al. compared the pressure resistance ability of stepped magnetic fluid seal with large clearance and ordinary magnetic fluid seal and numerically and experimentally studied the influences of sealing clearance and leakage path on the pressure 
resistance ability of stepped magnetic fluid seal. Yang and $\mathrm{Li}$ conducted experimental studies on the ability of ordinary magnetic fluid and stepped magnetic fluid $[10,11]$. However, there is a disadvantage of stepped magnetic fluid seal, which is that the number of axial sealing clearance is less than that of radial sealing clearance.

A new type of magnetic fluid sealing device with large clearance and dual magnetic sources is designed. The magnetic field distribution in the sealing clearance is obtained by finite element magnetic field analysis. According to the theory of divergent magnetic fluid seal, the theoretical value of sealing pressure resistance is calculated. The influences of key parameters such as the volume of magnetic fluid on the pressure resistance ability of divergent magnetic fluid seal are experimentally studied. The experimental value is compared with the theoretical value, which verifies the correctness of the pressure resistance theory of divergent magnetic fluid seal. It is of great significance to solve the problem of magnetic fluid seal under the condition of large clearance and the application and development of magnetic fluid sealing technology.

\section{Pressure Resistance Theory of Divergent Magnetic Fluid Seal}

In general, Bernoulli equation of magnetic fluid can be expressed by the following formula:

$$
P+\frac{1}{2} \rho_{f} V^{2}+\rho_{f} g h-\mu_{0} \int_{0}^{H} M d H=C,
$$

where $P$ is the pressure of magnetic fluid at some specific location; $h, \rho_{f}, V$, and $M$ are the height, density, velocity, and saturation magnetization of the magnetic fluid at some certain position, respectively; $g$ is the acceleration of gravity; $\mu_{0}$ is the vacuum permeability; $H$ is the external magnetic field strength; and $C$ is a constant. The effect of velocity on the magnetic fluid seal can be ignored, and the gravity effect in the sealing clearance can also be ignored. Therefore, the total sealing ability of magnetic seal is simplified as follows:

$$
\begin{aligned}
\Delta P & =\mu_{0} M_{S} \sum_{i=1}^{N}\left(H_{\max }^{i}-H_{\min }^{i}\right) \\
& =M_{S} \sum_{i=1}^{N}\left(B_{\max }^{i}-B_{\min }^{i}\right),
\end{aligned}
$$

where $H_{\max }^{i}$ and $H_{\min }^{i}$ represent the maximum and minimum magnetic field strengths under the $i$-th pole teeth, respectively; $B_{\max }^{i}$ and $B_{\min }^{i}$ represent the maximum and minimum flux densities under the $i$-th pole teeth, respectively; and $N$ is the total number of sealing magnetic pole. According to the pressure resistance theory of divergent magnetic fluid seal [11], the pressure resistance ability can be calculated as follows:

$$
\Delta P=\sum_{\mathrm{i}=1}^{\mathrm{n}}\left(p_{i r}+\delta p_{i \mathrm{a}}\right),
$$

where $P_{i r}$ is the pressure resistance ability of magnetic fluid seal within the height of radial sealing clearance formed by the $i$-th pole shoe and step shaft; and $P_{i a}$ is the pressure resistance ability of magnetic fluid seal within the width of axial sealing clearance formed by the $i$-th pole shoe and step shaft; $\delta$ is a constant, and its size is calculated by the following formula:

$$
\delta=\left\{\begin{array}{ll}
1 & \text { when } \mathrm{p}_{\mathrm{ir}}<p_{i a} \\
0 & \text { others } \mathrm{p}_{\mathrm{ir}} \geq p_{i a}
\end{array} .\right.
$$

\section{Numerical Analysis of Divergent Magnetic Fluid Seal with Dual Magnetic Sources}

3.1. Structure Design of Divergent Magnetic Fluid Seal with Dual Magnetic Sources. In the magnetic field finite element analysis of magnetic fluid seal, it is necessary to simplify the two-dimensional model properly, and the simplified model can still form a complete magnetic circuit, so as to achieve the sealing effect. The simplified model consists of three pole shoes, two permanent magnets, and a step shaft. Because the magnetic fluid sealing structure is axisymmetric, its two-dimensional physical model is only half of the magnetic fluid sealing structure, as shown in Figure 1. The structural parameters of divergent magnetic fluid seal with large clearance and dual magnetic source are shown in Table 1.

Using ANSYS software, combined with the data given in Table 1 and the material characteristics of step shaft, pole shoe, and permanent magnet, the structure shown in Figure 1 is simulated by finite element method. The material of step shaft and pole shoe is 45 steel, and the material of permanent magnet is $\mathrm{NdFeB}$. The $\mathrm{N} 38 \mathrm{H}$ type of $\mathrm{NdFeB}$ permanent magnet was used. The performance parameters are shown in Table 2.

The intelligent grid level is set to 1 , and the parallel boundary conditions of magnetic lines are applied to solve the problem. When selecting a magnetic material, not only the magnetic permeability of the material but also the processing and cost of the material itself must be considered. Therefore, the magnetic material selected in this article is 45 steel, which is easy to buy and has good magnetic permeability [12]. The magnetization curve of No. 45 steel is shown in Figure 2.

\subsection{Magnetic Field Distribution in the Clearance of Divergent Magnetic Fluid Seal with Dual Magnetic Sources}

3.2.1. Magnetic Force Line Distribution. The magnetic flux density field lines distribution of the magnetic fluid seal is shown in Figure 3.

It can be seen from Figure 3 that the magnetic force lines passing through the pole teeth are relatively dense. By comparing the magnetic force lines on the three pole shoes, it can be found that the magnetic force lines of the corresponding pole teeth on the middle pole shoe are the densest, because the middle pole shoe is affected by two permanent magnets at the same time. 


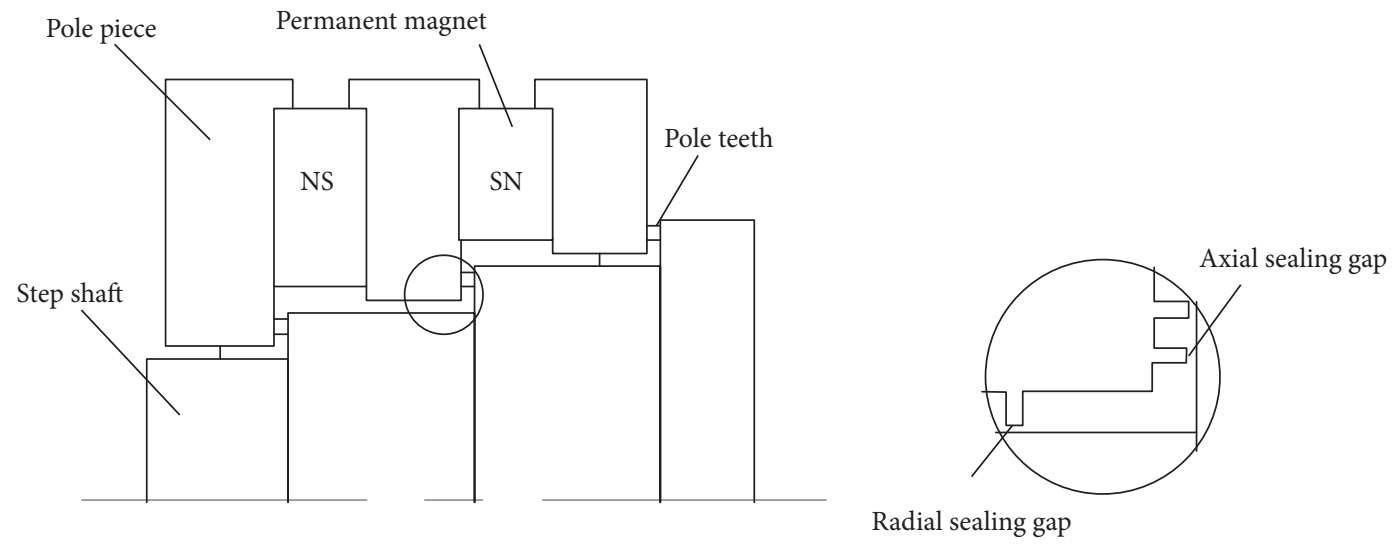

FIgURE 1: Structural diagram of divergent magnetic fluid seal with dual magnetic source.

TABLE 1: Structural parameters of divergent magnetic fluid seal with large clearance.

\begin{tabular}{lc}
\hline Parameters & Sizes \\
\hline Inner radius of the first pole shoe $(\mathrm{mm})$ & 14.1 \\
Inner radius of the second pole shoe $(\mathrm{mm})$ & 17.8 \\
Inner radius of the third pole shoe $(\mathrm{mm})$ & 21.5 \\
Outer radius of the first/second/third pole shoe $(\mathrm{mm})$ & 36 \\
Radius of the first step shaft $(\mathrm{mm})$ & $13.4 / 13.5 / 13.6 / 13.7$ \\
Step height $(\mathrm{mm})$ & 3.7 \\
Inner radius of the first stage permanent magnet $(\mathrm{mm})$ & 21.5 \\
Thickness of the first stage permanent magnet $(\mathrm{mm})$ & 6 \\
Inner radius of the second stage permanent magnet $(\mathrm{mm})$ & 22.5 \\
Thickness of the second stage permanent magnet $(\mathrm{mm})$ & 4.5 \\
Outer radius of the first/second stage permanent magnet $(\mathrm{mm})$ & 32.5 \\
Width of axial sealing clearance $(\mathrm{mm})$ & $0.4 / 0.5 / 0.6 / 0.7$ \\
Height of radial sealing clearance $(\mathrm{mm})$ & $0.4 / 0.5 / 0.6 / 0.7$ \\
Width of pole teeth $(\mathrm{mm})$ & 0.3 \\
Height of pole teeth $(\mathrm{mm})$ & 0.7 \\
\hline
\end{tabular}

TABLE 2: Magnetic property parameters.

\begin{tabular}{lc}
\hline Parameter & Numerical value \\
\hline$B_{r}(\mathrm{~T})$ & 1.227 \\
$H_{c b}(\mathrm{KA} / \mathrm{m})$ & 899 \\
$H_{c j}(\mathrm{KA} / \mathrm{m})$ & 1353 \\
Temperature $\left({ }^{\circ} \mathrm{C}\right)$ & 120 \\
\hline
\end{tabular}

3.2.2. Magnetic Field Distribution in the Clearance of Divergent Magnetic Fluid Seal with the Change of Teeth Number. When the axial and radial clearances are both $0.5 \mathrm{~mm}$, the axial pole teeth are 2 . The magnetic field distribution in the sealing clearance under different radial pole teeth numbers is shown in Figure 4, where 1, 2, 3, and 4 represent the numbers of radial pole teeth, respectively. When the axial clearance and radial clearance are both $0.5 \mathrm{~mm}$, the radial pole teeth is 2 . The magnetic field distribution in the sealing clearance under different axial pole teeth numbers is shown in Figure 5, where 1, 2, and 3 represent the numbers of axial pole teeth, respectively.

It can be seen from Figure 4 that with the increase of radial teeth, the number of magnetic flux density gradient in the radial sealing clearance of magnetic fluid seal is also increasing. However, the gradient difference of flux density corresponding to a single pole teeth is becoming smaller, which leads to the weakening of sealing ability. Therefore, a greater number of radial teeth does not mean that the sealing ability will become better.

As shown in Figure 5, with the increasing number of axial teeth, the total flux density difference in the axial sealing clearance width corresponding to each pole shoe in the magnetic fluid seal is also increasing, and the number of flux density gradient in the axial sealing clearance width is not equal to the number of axial pole teeth.

\subsubsection{Magnetic Field Distribution of Divergent Magnetic} Fluid Seal with the Change of Clearance in the Clearance. When the number of axial teeth and radial teeth are both 2 , and the axial clearance is $0.5 \mathrm{~mm}$, the magnetic field distribution in the sealing clearance under different radial clearance sizes is shown in Figure 6 , where $0.4 \mathrm{~mm}$, $0.5 \mathrm{~mm}, 0.6 \mathrm{~mm}$, and $0.7 \mathrm{~mm}$ are the heights of radial sealing clearance. When the number of axial teeth and radial teeth are both 2 , and the radial clearance is $0.5 \mathrm{~mm}$, the magnetic field distribution in the sealing clearance 


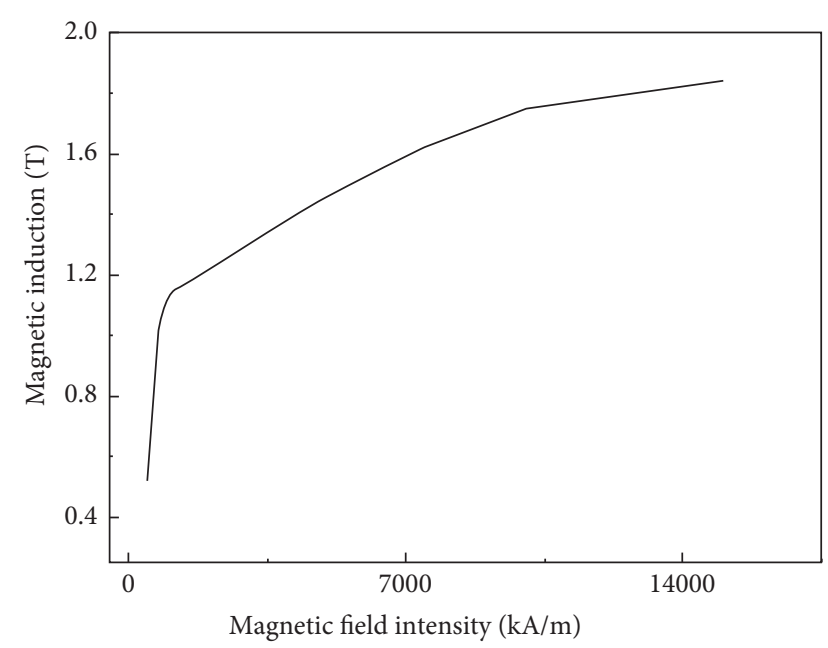

FIgURE 2: Magnetization curve of \#45 steel.

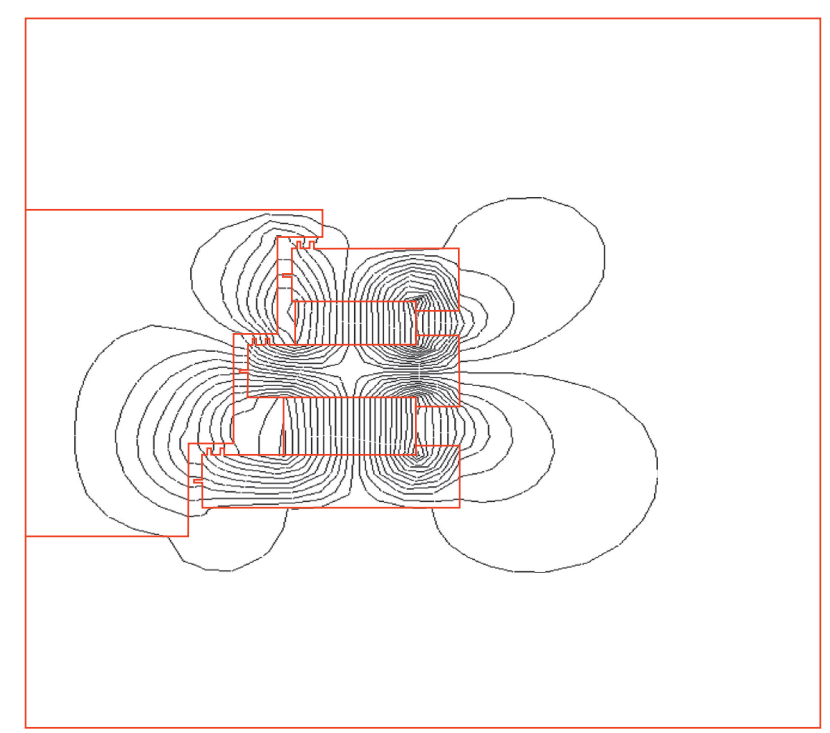

Figure 3: Distribution of magnetic flux density field lines of magnetic fluid seal.

under different axial clearance sizes is shown in Figure 7, where $0.4 \mathrm{~mm}, 0.5 \mathrm{~mm}, 0.6 \mathrm{~mm}$, and $0.7 \mathrm{~mm}$ are the heights of axial sealing clearance.

As indicated in Figure 6, with the increase of radial clearance, the magnetic flux density in the magnetic fluid sealing clearance will decrease. The reason for this phenomenon is that the magnetic resistance in the radial clearance is also increasing due to the increase of the radial clearance, which leads to the decrease of the magnetic flux density in the radial sealing clearance.

It can be seen from Figure 7 that with the increase of axial clearance, the magnetic flux density in the magnetic fluid sealing clearance will decrease. The reason for this phenomenon is that the magnetic resistance in the axial clearance increases with the increase of the axial clearance, which leads to the decrease of the magnetic flux density in the axial sealing clearance.
3.2.4. Magnetic Field Distribution in Divergent Magnetic Fluid Sealing Clearance under Different Shaft Eccentricity. The ratio of eccentricity to minimum end radius of stepped shaft is defined as eccentricity in the study of magnetic field distribution in sealing clearance under shaft eccentricity. When the axial clearance and radial clearance are $0.5 \mathrm{~mm}$ and the number of axial and radial teeth are both 2 , the magnetic field distribution in the sealing clearance under different shaft eccentricity is studied. When the shaft is eccentric, the magnetic field distribution of the larger side and the smaller side of the radial clearance is shown in Figures 8 and 9 , where $0,0.1 / 13.6,0.2 / 13.6$, and $0.3 / 13.6$ are the eccentricity of the rotating shaft.

As indicated in Figure 8, the magnetic flux density gradient on the larger side of the radial clearance decreases with the increase of eccentricity, which may be due to the increase of eccentricity, the increase of radial clearance height, and the increase of magnetic reluctance in the corresponding clearance, resulting in the decrease of magnetic flux density. It can also be seen from the figure that the magnetic flux density increases with the increase of rotating shaft eccentricity, and the step height decreases with the increase of rotating shaft eccentricity, so the step height also affects the magnetic field distribution of the magnetic fluid seal.

As shown in Figure 9, the magnetic flux density gradient of the smaller side of the radial clearance increases with the increase of eccentricity. As the eccentricity increases, the corresponding magnetic reluctance on the side with smaller radial clearance decreases, which leads to the increase of magnetic flux density. It can also be seen from the figure that the magnetic flux density decreases with the increase of the rotating shaft eccentricity. This is because the step height increases with the increase of rotating shaft eccentricity at the position where the clearance is small.

\section{Experiment Process and Method}

The pressure resistance test of divergent magnetic fluid seal and ordinary magnetic fluid seal is carried out on the test bench of magnetic fluid seal, as shown in Figure 10. The test bench is composed of magnetic fluid seals, pressure gauge, nitrogen cylinder, sealing cavity, and motor.

The experimental steps are as follows: firstly, the shell is heated by the high and low temperature test chamber, and then the thermal assembly is carried out. After the installation, the magnetic fluid seals are put into the test chamber with high and low temperatures to reduce its temperature, so as to prevent the magnetic fluid from losing its function due to too high temperature. Then, the sealing components and the sealing cavity are connected by screws, and finally the motor and the rotating shaft are connected by the coupling.

In the experiment, the motor drives the step shaft to rotate through the coupling and pressurizes the seals through the nitrogen cylinder. When the pressure is placed, we should pay attention to the pressure gauge and spray the foam water with the watering pot at the end cover of the sealing assembly. If the sealing assembly leaks, the bubble will appear at the end cover of the sealing component, and 


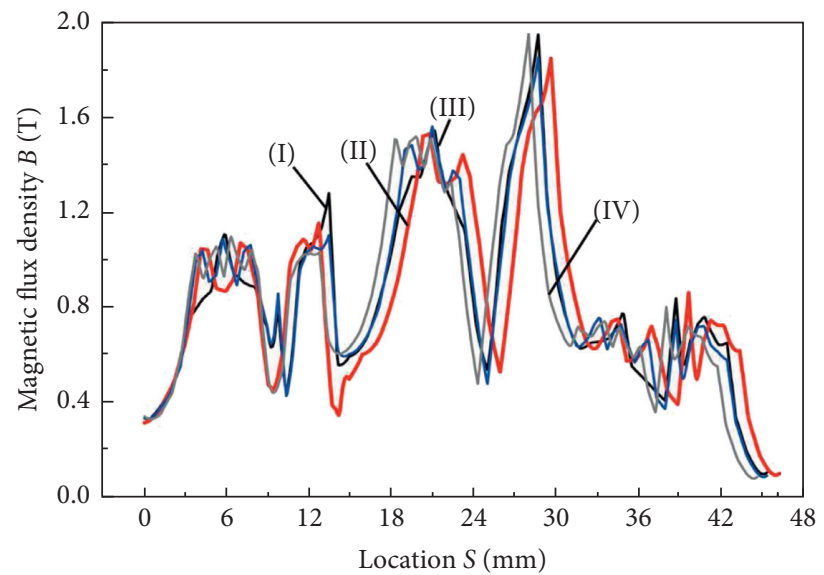

Figure 4: Magnetic field distribution in magnetic fluid sealing clearance with different radial teeth numbers (I), 1; (II), 2; (III), 3; and (IV), 4.

we record the pressure value at this time. This value is the maximum sealing pressure resistance ability. After the test, the sealing assembly is disassembled and cleaned with highpressure water gun. After cleaning, the next group of experiments is carried out.

\section{Experimental Results and Discussion}

5.1. Influence of Magnetic Fluid Injection Volume. To a certain extent, the volume of magnetic fluid injection will affect the pressure resistance ability of the magnetic fluid seal. Therefore, when we study the pressure resistance ability of the magnetic fluid seal, we need to study the injection volume first and get the saturation magnetic fluid injection volume required by the experiment. When the clearance is the largest and the number of teeth is the largest, the influence of magnetic fluid injection on the pressure resistance of stepped magnetic fluid seal is shown in Figure 11.

It can be seen from Figure 11 that with the increase of magnetic fluid injection volume, the pressure resistance ability of magnetic fluid seal is also increasing. When the volume of magnetic fluid injection exceeds $3 \mathrm{ml}$, the sealing pressure resistance ability does not increase greatly and tends to be stable gradually. Therefore, the saturated magnetic fluid injection volume of divergent magnetic fluid seal is $3 \mathrm{ml}$.

5.2. Influence of the Number of Pole Teeth on the Pressure Resistance Ability of Divergent Magnetic Fluid Seal. When the axial and radial clearance is $0.5 \mathrm{~mm}$, the axial teeth number is 2 , and the injection volume of magnetic fluid is $3 \mathrm{ml}$, the influence of radial teeth number on the pressure resistance ability of divergent magnetic fluid seal and ordinary magnetic fluid seal is shown in Figure 12. When the axial and radial clearance is $0.5 \mathrm{~mm}$, the radial teeth number is 2 , and the injection volume of magnetic fluid is $3 \mathrm{ml}$, the influence of axial teeth number on the pressure resistance ability of divergent magnetic fluid seal and ordinary magnetic fluid seal is shown in Figure 13.

It can be seen from Figure 12 that with the increase of radial teeth number, the experimental value of the divergent magnetic fluid seal first increases and then decreases. This is consistent with the theoretical value of the sealing pressure resistance obtained by the numerical simulation of the magnetic field finite element method, which shows that the two are in good agreement. It can be seen from the figure that the pressure resistance experimental value of the divergent magnetic fluid seal is far greater than that of the ordinary magnetic fluid seal, and when the radial teeth number is 2 , the pressure resistance experimental value is 6 times of the ordinary experimental value.

As shown in Figure 13, with the increase of axial teeth number, the pressure resistance value of divergent magnetic fluid seal is also increasing, which is consistent with the theoretical value of divergent magnetic fluid seal when the axial pole teeth are changed through the numerical simulation of magnetic field finite element method, which shows that the two are in good agreement. It can be seen from the figure that the experimental value of the ordinary magnetic fluid seal is a straight line, because there is no axial clearance in ordinary magnetic fluid seal. When the number of axial teeth is 3 , the pressure resistance value is 6 times of that of ordinary magnetic fluid.

5.3. Influence of Sealing Clearance on Pressure Resistance Ability of Divergent Magnetic Fluid Seal. When the numbers of axial and radial teeth are 2, the axial clearance is $0.5 \mathrm{~mm}$, and the injection volume of magnetic fluid is $3 \mathrm{ml}$, the influence of radial clearance on the pressure resistance ability of divergent magnetic fluid seal and ordinary magnetic fluid seal is shown in Figure 14. When the numbers of axial and radial teeth are 2 , the radial clearance is $0.5 \mathrm{~mm}$, and the injection volume of magnetic fluid is $3 \mathrm{ml}$, the influence of axial clearance on the pressure resistance ability of divergent magnetic fluid seal and ordinary magnetic fluid seal is shown in Figure 15.

As indicated in Figure 14, with the increase of radial clearance, the pressure resistance value of divergent magnetic fluid seal first increases and then decreases, which is consistent with the theoretical value of pressure resistance of divergent magnetic fluid seal obtained by magnetic field finite element method simulation, which shows that the two 


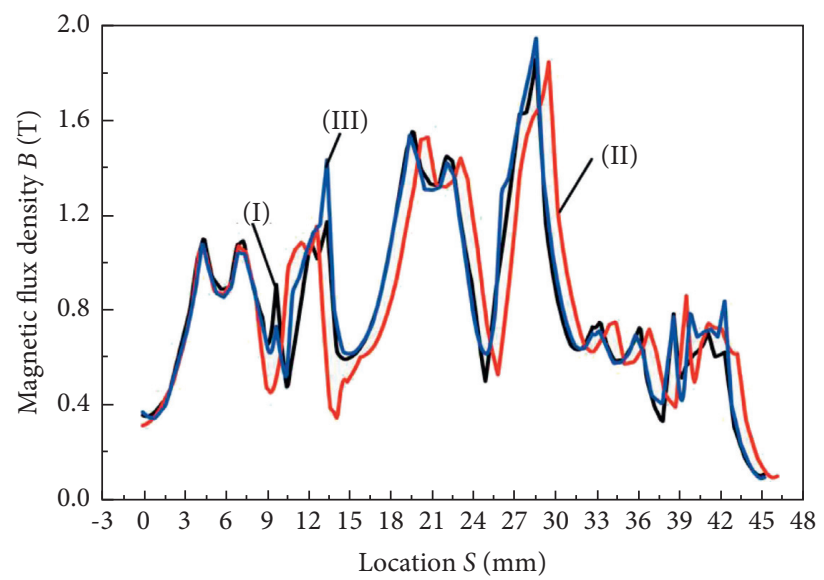

Figure 5: Magnetic field distribution in magnetic fluid sealing clearance with different axial teeth numbers (I), 1; (II), 2 ; and (III), 3.

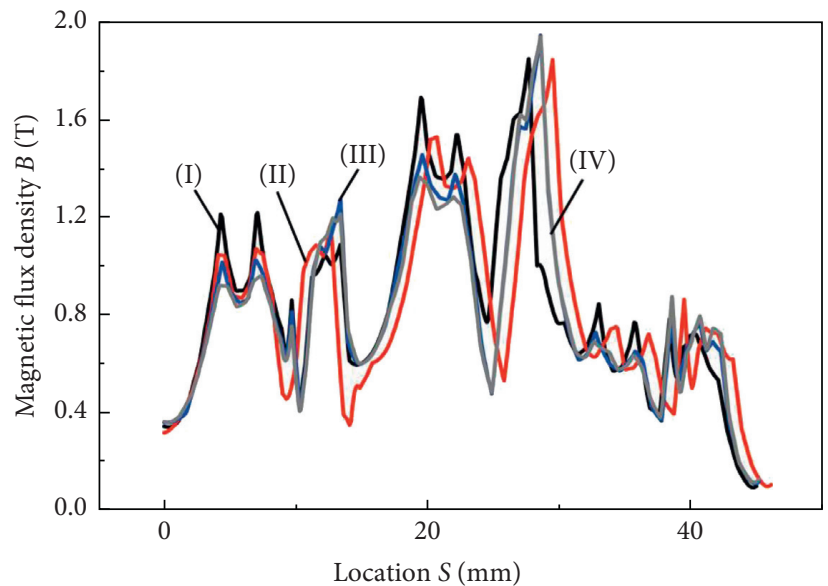

FiguRE 6: Magnetic field distribution in magnetic fluid sealing clearance under different radial clearances (I), $0.4 \mathrm{~mm}$; (II), $0.5 \mathrm{~mm}$; (III), $0.6 \mathrm{~mm}$; and (IV), $0.7 \mathrm{~mm}$.

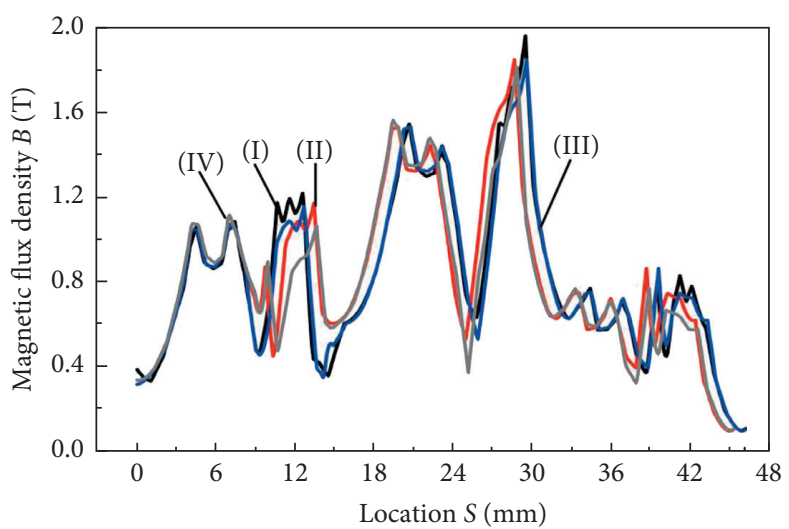

Figure 7: Magnetic field distribution in magnetic fluid sealing clearance under different axial clearances (I), $0.4 \mathrm{~mm}$; (II), $0.5 \mathrm{~mm}$; (III), $0.6 \mathrm{~mm}$; and (IV), $0.7 \mathrm{~mm}$.

are in good agreement. It can also be seen from the figure that when the radial clearance is $0.4 \mathrm{~mm}$, the pressure resistance value is about 4 times of that of the ordinary sealing test.
It can be seen from Figure 15 that with the increase of axial clearance, the pressure resistance value of divergent magnetic fluid seal decreases. When the axial clearance increases to $0.6 \mathrm{~mm}$, the pressure resistance theoretical value 


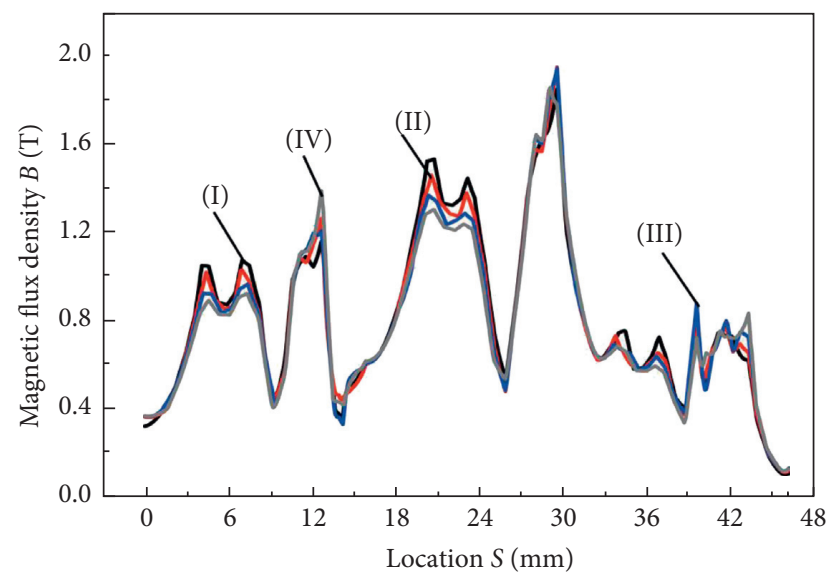

FIGURE 8: Distribution of magnetic field in larger radial clearance under different eccentricity of rotating shaft (I), 0; (II), 0.1/13.6; (III), 0.2/ 13.6; (IV), 0.3/13.6.

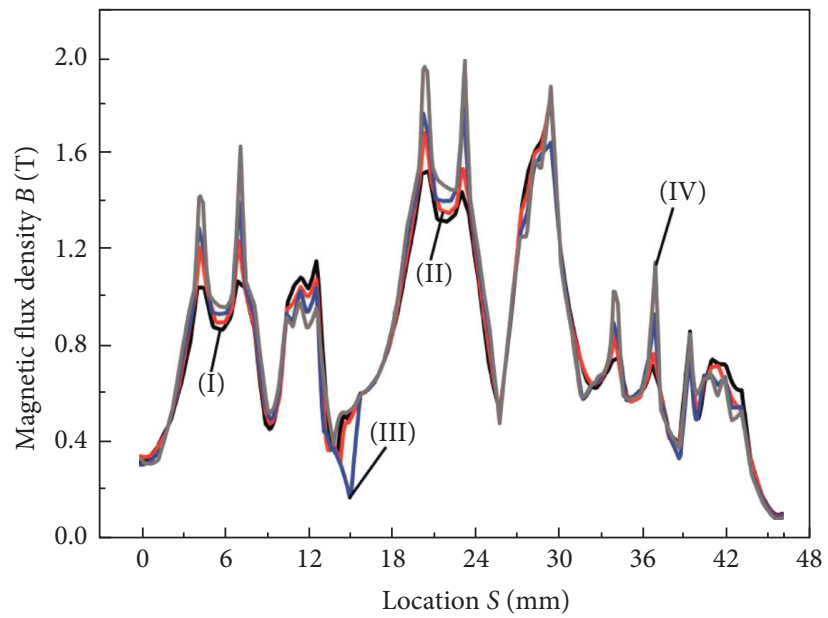

FIGURE 9: Distribution of magnetic field in smaller radial clearance under different eccentricity of rotating shaft (I), 0; (II), 0.1/13.6; (III), 0.2/ 13.6; and (IV), 0.3/13.6.

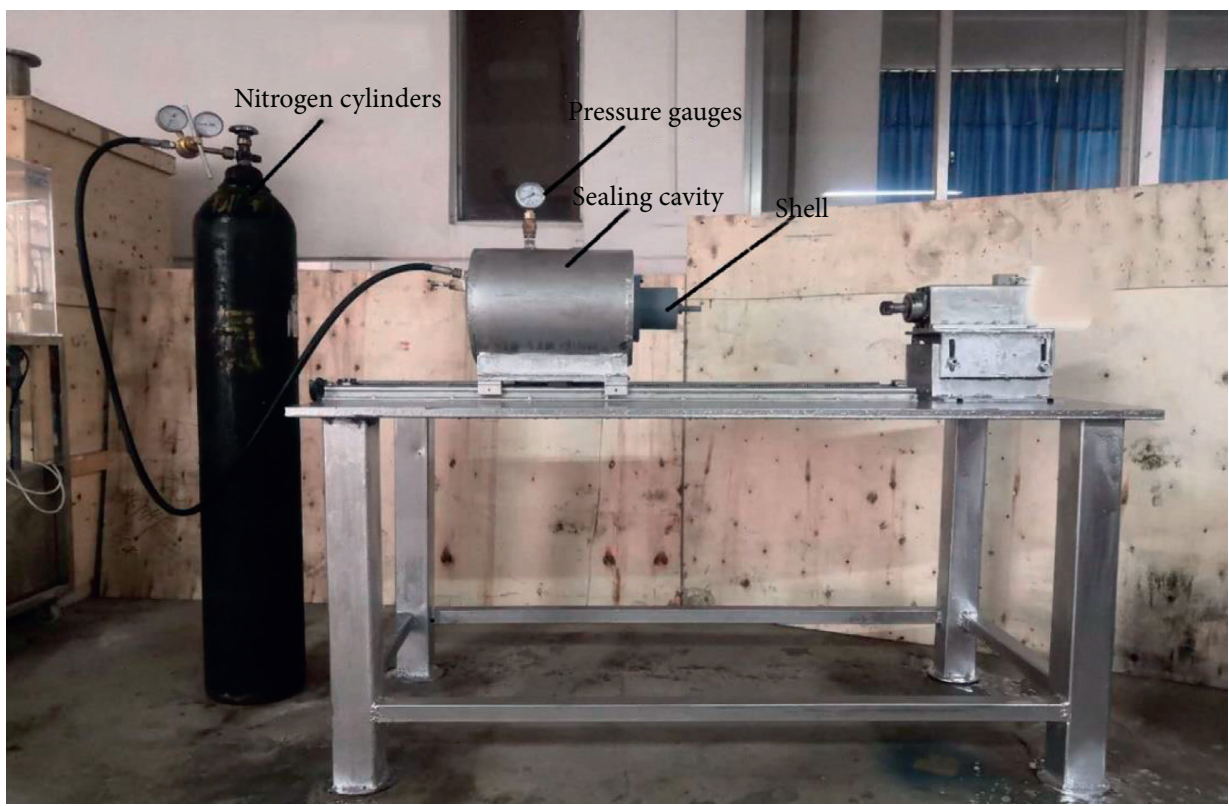

Figure 10: Test bench of magnetic fluid seal. 


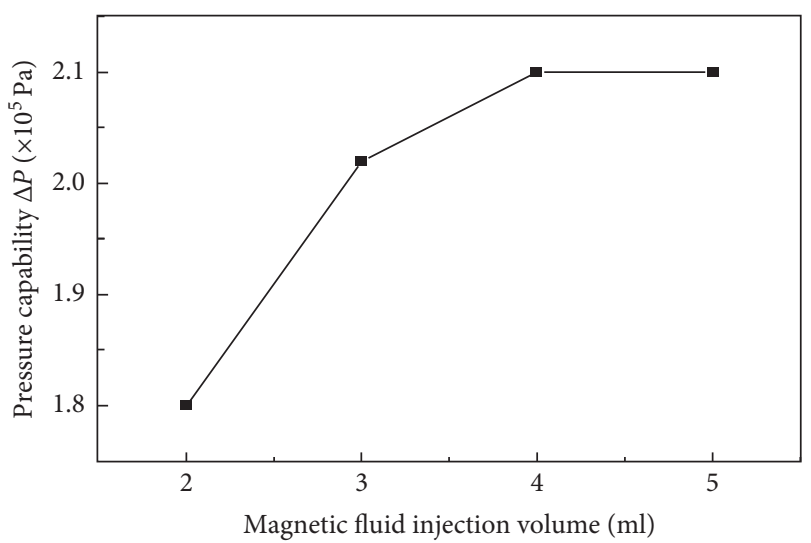

FIGURE 11: Influence of volume injection on magnetic fluid sealing ability.

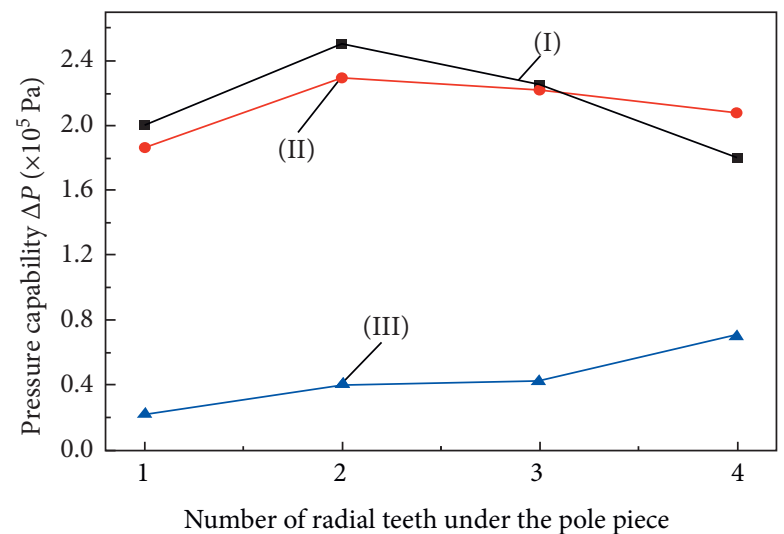

Figure 12: Influence of radial teeth number on pressure resistance ability of magnetic fluid seal. (I) Experimental value of divergent magnetic fluid seal. (II) Theoretical value of divergent magnetic fluid seal. (III) Experimental value of ordinary magnetic fluid seal.

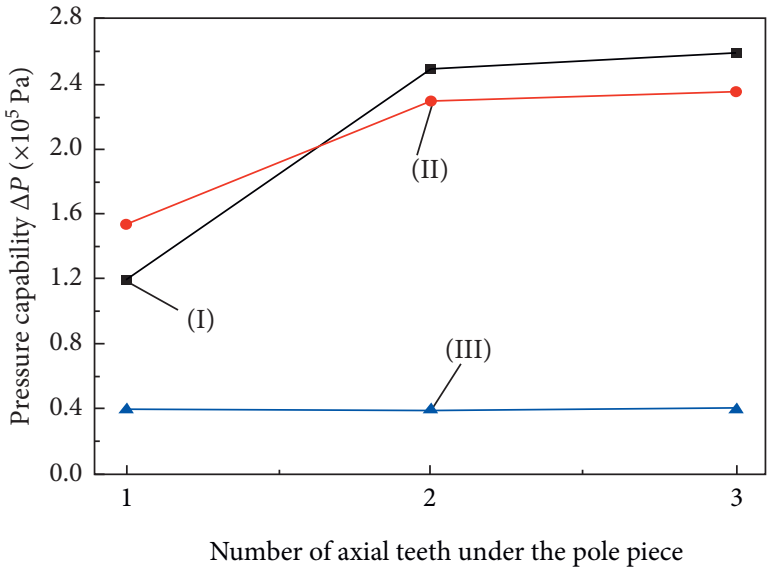

FIGURE 13: Influence of axial teeth number on pressure resistance ability of magnetic fluid seal. (I) Experimental value of divergent magnetic fluid seal. (II) Theoretical value of divergent magnetic fluid seal. (III) Experimental value of ordinary magnetic fluid seal. 


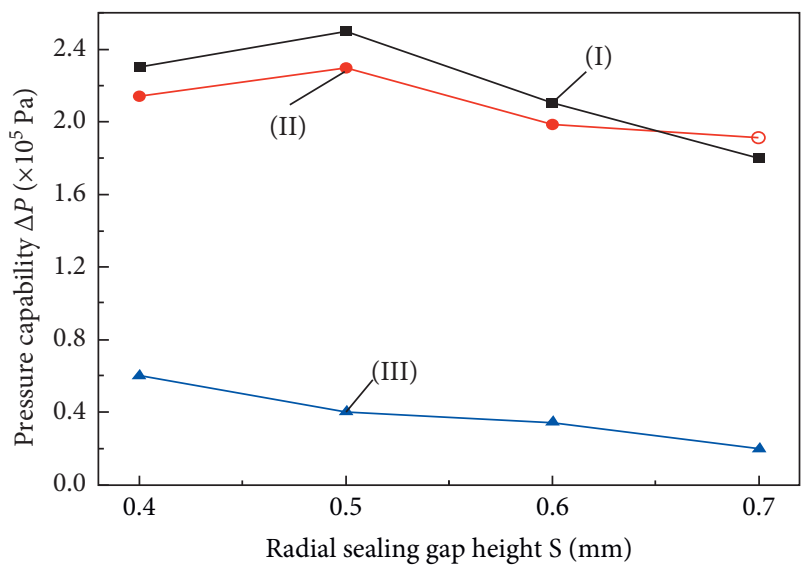

FIGURE 14: Influence of radial clearance on pressure resistance ability of magnetic fluid seal. (I) Experimental value of divergent magnetic fluid seal. (II) Theoretical value of divergent magnetic fluid seal. (III) Experimental value of ordinary magnetic fluid seal.

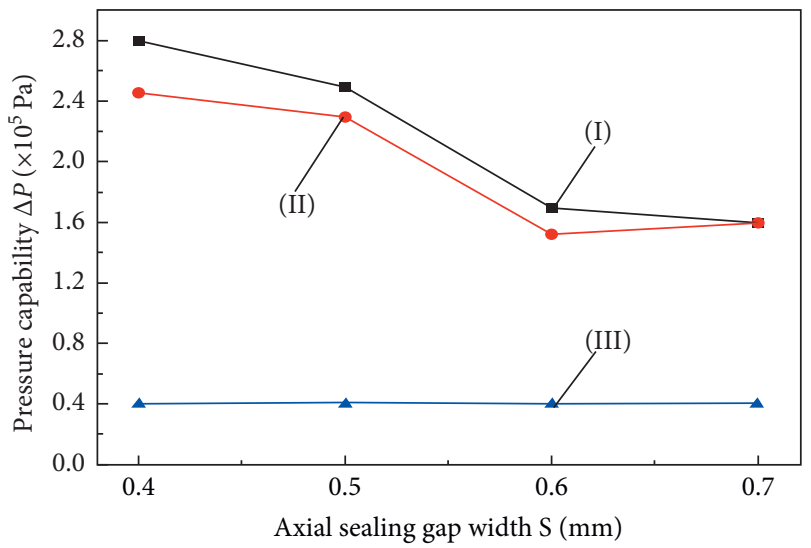

FIGURE 15: Influence of axial clearance on pressure resistance ability of magnetic fluid seal. (I) Experimental value of divergent magnetic fluid seal. (II) Theoretical value of divergent magnetic fluid seal. (III) Experimental value of ordinary magnetic fluid seal.

and pressure resistance experimental value of the divergent magnetic fluid seal tend to be stable, which shows that the two are in good agreement. It can be seen from the figure that the pressure resistance experimental value of ordinary magnetic fluid seal is a straight line, because there is no axial clearance in the ordinary type. When the axial clearance is $0.4 \mathrm{~mm}$, the pressure resistance experimental value of divergent magnetic fluid seal is 7 times of that of ordinary magnetic fluid seal.

5.4. Influence of Rotating Shaft Eccentricity on Pressure Resistance Ability of Divergent Magnetic Fluid Seal. When the numbers of axial and radial teeth are 2 , the axial clearance and radial clearance are $0.5 \mathrm{~mm}$, and the injection volume of magnetic fluid is $3 \mathrm{ml}$, the influence of rotating shaft eccentricity on the pressure resistance ability of divergent magnetic fluid seal is shown in Figure 16. The sealed shaft of the eccentricity is defined as the ratio of the distance between the two-axes line to the minimum radius of the stepped shaft.

It can be seen from Figure 16 that with the increase of rotating shaft eccentricity, the experimental value of pressure resistance of divergent magnetic fluid seal decreases, which is consistent with the result of finite element numerical simulation. The eccentricity of rotating shaft has a great influence on the experimental value and theoretical value of pressure resistance of divergent magnetic fluid seal, so the influence of eccentricity should be avoided as far as possible. 


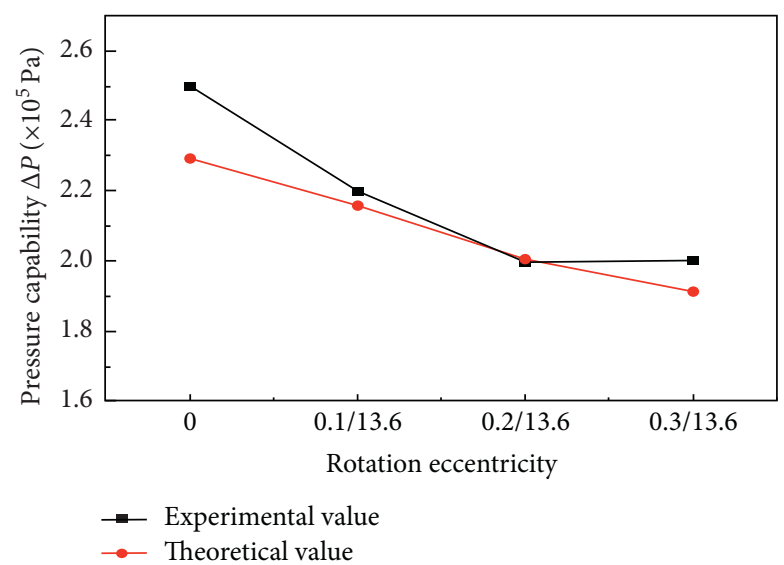

FIGURE 16: Influence of rotating shaft eccentricity on pressure resistance ability of divergent magnetic fluid seal.

\section{Conclusion}

A structure of divergent magnetic fluid seal is designed, and the magnetic field distribution in the clearance of the twodimensional divergent magnetic fluid seal structure is numerically simulated by using the magnetic field finite element method. According to the theoretical formula of sealing pressure resistance, the corresponding theoretical value of pressure resistance is calculated. The influences of the volume of magnetic fluid injection, the number of radial and axial pole teeth, height of radial seal clearance and width of axial seal clearance, and the eccentricity of rotating shaft on the pressure resistance ability of divergent magnetic fluid seal were studied experimentally. The experimental results were compared and analyzed with its theoretical values of pressure resistance. The conclusions are as follows:

(1) Under the condition of large clearance, the saturated injection volume of magnetic fluid in the divergent magnetic fluid sealing clearance with secondary magnetic source is $3 \mathrm{ml}$

(2) Pressure resistance experimental value of divergent magnetic fluid seal with secondary magnetic source and large clearance fits well with the theoretical value of that

(3) The pressure resistance capability of divergent stepped magnetic fluid seal is significantly better than that of ordinary magnetic fluid seal

(4) In order to obtain better sealing capability, the number of radial pole teeth should be less than that of axial pole teeth, and the height of radial sealing clearance should be less than the width of axial sealing clearance when designing divergent stepped magnetic fluid seal

\section{Data Availability}

Experimental data are provided within the text.

\section{Conflicts of Interest}

The authors declare that they have no conflicts of interest.

\section{Acknowledgments}

The authors gratefully acknowledge the support of the National Nature Science Foundation of China (Grant no. 51905114), Science and Technology Project of Guangxi Province (Grant no. 2020GXNSFAA159042), Science and Technology Project of Liuzhou (Grant no. 2017BC20204), and Innovation Project of Guangxi University of Science and Technology Graduate Education (Grant no. GKYC202111).

\section{References}

[1] M. Cong and H. Shi, "A study of magnetic fluid rotary seals for wafer handling robot," in Proceedings of the 2008 15th International Conference on Mechatronics and Machine Vision in Practice, pp. 158-170, Auckland, New Zealand, 2008.

[2] M. S. Sarma, P. Stahl, and A. Ward, "Magnetic-field analysis of ferrofluidic seals for optimum design," Journal of Applied Physics, vol. 55, no. 6, pp. 2595-2597, 1984.

[3] L. Matuszewski, "Failure of magnetic fluid seals operating in water: preliminary conclusions," Polish Maritime Research, vol. 24, no. 2, pp. 113-120, 2017.

[4] M. Kroell, M. Pridoehl, G. Zimmermann, L. Pop, S. Odenbach, and A. Hartwig, "Magnetic and rheological characterization of novel ferrofluids," Journal of Magnetism and Magnetic Materials, vol. 289, pp. 21-24, 2005.

[5] M. Szczech and W. Horak, "Numerical simulation and experimental validation of the critical pressure value in ferromagnetic fluid seals," IEEE Transactions on Magnetics, vol. 53, no. 7, pp. 1-5, 2017.

[6] Z. Meng, Z. Jibin, and H. Jianhui, "An analysis on the magnetic fluid seal capacity," Journal of Magnetism and Magnetic Materials, vol. 303, no. 2, pp. e428-e431, 2006.

[7] M. Szczech, "Experimental study on the pressure distribution mechanism among stages of the magnetic fluid seal," IEEE Transactions on Magnetics, vol. 54, no. 6, pp. 1-7, 2018.

[8] S. Parmar, V. Ramani, R. V. Upadhyay, and K. Parekh, "Design and development of large radial clearance static and dynamic magnetic fluid seal," Vacuum, vol. 156, pp. 325-333, 2018.

[9] S. Parmar, V. Ramani, R. V. Upadhyay, and K. Parekh, "Two stage magnetic fluid vacuum seal for variable radial clearance," Vacuum, vol. 172, Article ID 109087, 2020. 
[10] X. L. Yang and D. C. Li, "Experimental investigation of diverging stepped magnetic fluid seals with large sealing gap," International Journal of Applied Electromagnetics and Mechanics, vol. 50, no. 3, pp. 407-415, 2016.

[11] X. Yang, P. Sun, F. Chen, F. Hao, D. Li, and P. J. Thomas, "Numerical and experimental studies of a novel converging stepped ferrofluid seal," IEEE Transactions on Magnetics, vol. 55, no. 3, pp. 1-6, 2019.

[12] J. Huang, Y. Zhou, and X. Wang, "Study on braking torque of disc type and cylinder type magnetorheological fluid brake," Machinery Design And Manufacture, vol. 4, pp. 71-74, 2020. 